
\title{
CS Research Square \\ Linking emotional valence and anxiety in a mouse insula-amygdala circuit
}

\section{Céline Nicolas}

University of Bordeaux, Neurocentre Magendie, INSERM 1215

Anes Ju

Bordeaux University

\section{Yifan Wu}

University of Bordeaux, Neurocentre Magendie, INSERM 1215

Hazim Eldirdiri

University of Bordeaux, Neurocentre Magendie, INSERM 1215

\section{Sebastien Delcasso}

University of Bordeaux 1

\section{Débora Jacky}

University of Bordeaux, Neurocentre Magendie, INSERM 1215

\section{Laura Supiot}

University of Bordeaux, Neurocentre Magendie, INSERM 1215

\section{Claudia Fornari}

University of Bordeaux, Neurocentre Magendie, INSERM 1215

\section{Adrien Vérité}

University of Bordeaux, Neurocentre Magendie, INSERM 1215

\section{Maxime Masson}

University of Bordeaux, Neurocentre Magendie, INSERM 1215

\section{Silvia Rodriguez-Rozada}

University Medical Center Hamburg-Eppendorf

\section{J. Simon Wiegert}

University Medical Center Hamburg-Eppendorf https://orcid.org/0000-0003-0893-9349

\section{Anna Beyeler ( $\nabla$ annabey@gmail.com )}

University of Bordeaux https://orcid.org/0000-0003-2371-5706

\section{Article}

Keywords: emotional valence, anxiety, insula-amygdala circuit

Posted Date: October 26th, 2021 
DOl: https://doi.org/10.21203/rs.3.rs-964107/v1

License: (c) (1) This work is licensed under a Creative Commons Attribution 4.0 International License. Read Full License 


\section{Abstract}

The response of the insular cortex (IC) and amygdala to stimuli of positive and negative valence were found to be altered in patients with anxiety disorders. However, the coding properties of neurons controlling anxiety and valence remain unknown. Combining photometry recordings and chemogenetics in mice, we uncover the anxiogenic control of projection neurons in the anterior IC (aIC), independently of their projection target. Using viral tracing and ex vivo electrophysiology, we characterize the monosynaptic alC to the basolateral amygdala (BLA) connection, and employed projection-specific optogenetics, to reveal anxiogenic properties of aIC-BLA neurons in anxiety-related behaviors. Finally, using photometry recordings, we identified that aIC-BLA neurons are active in anxiogenic spaces, and in response to aversive stimuli. Together, these findings show that negative valence, as well as anxietyrelated information and behaviors, are encoded by aICBLA glutamatergic neurons, providing a starting point to understand how alterations of this pathway contribute to psychiatric disorders.

\section{Introduction}

Anxiety is defined as the anticipation of a future threat, with an uncertain probability of occurrence $e^{1-3}$. Importantly, anxiety is a physiological and adaptive state, evolutionarily relevant, since it allows organisms to prevent exposure to harmful situations. Anxiety becomes pathological when avoidance behaviors and fear are sustained and disruptive despite the absence of danger or potential danger ${ }^{1,4}$. Clinical studies demonstrated that patients with anxiety disorders have altered attribution of emotional valence, as they exhibited an attentional bias for stimuli of negative valence, as well as an increase in negative interpretations of ambiguous sentences and scenarios compared to healthy controls ${ }^{5-7}$. Consequently, it has been hypothesized for almost a decade that the neural circuits encoding anxiety and emotional valence overlap. In this regard, the insular cortex (IC, also named insula) is a key structure, as it has been shown to be involved in both valence processing and anxiety disorders ${ }^{8}$.

A functional imaging study has revealed that, in healthy individuals, the insula exhibits opposing responses to stimulations of negative and positive valence, compared to neutral stimulations, with higher activity in response to aversive stimuli, and lower activity in response to rewarding stimulations ${ }^{9}$. Interestingly, this study, along with a meta-analysis of a dozen of other imaging studies, identified hyperactivity of the insula in patients with anxiety disorders compared to healthy controls, in response to stimulations of negative valence ${ }^{9-12}$. Preclinical studies have confirmed the implication of the insula in valence- and anxiety-related behaviors. Interestingly, according to the antero-posterior axis, the insula has been shown to have opposite functions on positive and negative valence as well as anxiety-related behaviors, highlighting a functional dichotomy along its rostro-caudal axis. Specifically, optogenetic activation of excitatory neurons of the anterior or posterior insular cortices (aIC or pIC) promotes approach and avoidance behaviors respectively, suggesting the contribution of alC in positive valence and $\mathrm{pIC}$ in negative valence ${ }^{13,14}$. However, a pharmacological study including both activation and 
inhibition of these insular regions during the elevated plus maze test (EPM) in mice, demonstrated that the aIC has anxiogenic properties, whereas the pIC has anxiolytic properties ${ }^{15}$.

Multiple imaging studies, including a meta-analysis, also identified the amygdala as a crucial player in patients with anxiety disorders ${ }^{11}$. In addition, the amygdala was shown to mediate valence processing, as it is responding to stimuli of positive and negative valence in healthy individuals ${ }^{16}$. Interestingly, in patients with anxiety disorders, the amygdala is hyperactivated in response to images of negative valence $^{12}$. Consistently, preclinical studies have reported that projection neurons of the basolateral amygdala (BLA) control anxiety-related behaviors ${ }^{17,18}$, and that distinct neuronal populations of the BLA mediate positive and negative valence ${ }^{19-21}$.

Alteration of the functional connectivity between the insula and the amygdala has been reported in patients with anxiety disorders, highlighting crucial contribution of this connection in pathological anxiety ${ }^{11,22,23}$. Anatomical connections between these two regions have been described in mice ${ }^{24-27}$, and optogenetic activation of alC inputs in the BLA imposes positive valence to a neutral stimulus, while reversing the aversive value of a bitter tastant ${ }^{27}$. Moreover, most aIC-BLA neurons (80\%) express serotonin receptors ${ }^{28}$. Based on the fact that the serotonergic system is a pharmaceutical target of pathological anxiety ${ }^{29}$, this suggests a contribution of alC-BLA projection neurons in anxiety. However, the functional role of aIC-BLA neurons in anxiety remained unexplored. Using multifaceted circuit dissection in mice, including calcium imaging, anterograde tracing, electrophysiological mapping ex vivo, as well as chemo- and opto-genetic manipulations, we identified an anxiogenic role of alC glutamatergic neurons, and aIC-BLA neurons, along with an activation of aIC-BLA neurons during negative valence processing.

\section{Results}

\section{Anterior but not posterior glutamatergic insular neurons increase their activity in anxiogenic spaces}

To investigate how aIC and pIC are involved in anxiety, we recorded the activity of projection neurons in these cortical regions during anxiety-related behaviors with fiber photometry, expressing GCaMP6f under the CaMKII promoter (Fig. 1a,b and Extended data Fig. 1a-b,j,k). In the elevated plus maze test (EPM), an increase of the global calcium signal in alC glutamatergic neurons was detected during the exploration of the open arms, in comparison to the closed arms (Fig. 1c). Similarly, during the open field test (OFT), the same neural population exhibited an increase of calcium signal in the center compared to the borders of the arena (Fig. 1e), showing that glutamatergic neurons in alC are more active during the exploration of anxiogenic spaces (open arms of the EPM and center of the OFT). This increase in activity was independent of locomotion, as the velocity was the same in the open and closed arms (Extended data Fig. 1g), indicating that the mouse location in the EPM, which affects the anxiety state, might be a defining factor of the activity of alC excitatory neurons. Thus, we plotted the calcium signal depending on the mouse location within the open arms (Extended data Fig. 1h), and depending on the movement 
direction of mice in the open arms: when mice went out to explore the open arm (OUT), or went back towards the closed arms (IN). Interestingly, the activity of alC glutamatergic neurons was higher when mice were in the center compared to the extremity of the open arms, specifically while going out in the open arms (Extended data Fig. 1i). In contrast, glutamatergic neurons in the pIC exhibited comparable amount of neural activity when the mice were located in the anxiogenic and safe spaces of the EPM (Fig. 1d) and OFT (Fig. 1f), although the overall level of anxiety of these mice was similar to mice in the alC group (same time spent in anxiogenic spaces, Extended data Fig. 1e,f). These results suggest that glutamatergic neurons in the alC selectively encode anxiogenic spaces.

Thus, we hypothesized that inhibition of alC glutamatergic neurons will reduce anxiety-related behaviors. We used a chemogenetic approach (Fig. 1g) to inhibit the activity of alC glutamatergic neurons by expressing hM4Di, under the CaMKII promoter. The inhibition of these neurons during anxiety tests resulted in an increase in time mice spent in the open arms of EPM (Fig. 1h) and center of the OFT (Fig. 1k), during the second half of the test, in comparison to control mice. Importantly, inhibition of alC glutamatergic neurons did not affect the total distance travelled (Fig. 1i,I), or the locomotion speed (Fig. 1j,m). Taken together, these results show that activity of glutamatergic neurons of the alC encode anxiogenic spaces and control the level of anxiety-related behaviors.

\section{Posterior insula glutamatergic neurons are active in response to the consumption of an aversive tastant}

Previous studies suggested that neurons in the aIC and pIC are involved in emotional valence processing ${ }^{14}$, especially for the positive and negative valence of gustatory information, respectively. Thus, we performed fiber photometry recordings of alC and pIC glutamatergic neurons during sucrose (Fig. 2a) and quinine (Fig. 2b) consumption to evaluate their neural dynamics in response to stimuli of positive and negative valence. Peri-licking analysis of the calcium signal showed there was no changes of calcium signal between the baseline and post-lick periods in glutamatergic neurons of the alC and pIC (Fig. 2bd). Contrarily, a rapid increase of the global calcium signal was detected in the pIC after mice licked quinine, compared to the pre-licking baseline (Fig. $2 \mathrm{~h}$ ), whereas no changes were observed in alC glutamatergic neurons (Fig. 2g).

\section{The alC mainly projects to the BLA and the pIC to the central amygdala (CeA)}

To map the density of long-range projections of glutamatergic neurons of the alC and pIC to other key brain regions involved in anxiety or valence, we virally expressed eYFP under the CaMKII promoter in glutamatergic neurons of the alC or pIC to label their cell bodies, dendrites and axonal projections (Fig. 3a). After 4 weeks of expression, eYFP fluorescence was quantified in twelve downstream regions (Fig. 3b,c) and normalized to the region with the highest fluorescence intensity. Notably, the densest axonal fibers from the aIC and pIC were detected in two subdivisions of the amygdala; the BLA for alC projections, and the CeA, including the lateral and medial divisions (CeL and CeM) for pIC projections 
(Fig. 3d,e). However, these projections are not selective, as a substantial amount of axonal fibers from the alC were also detected in the CeL and CeM, and axonal fibers from the pIC were also detected in the BLA, which challenges the notion of two segregated insula-amygdala pathways (aIC-BLA and pIC-CeA) ${ }^{27}$. In addition, we identified strong and selective contralateral projection from the right to left alC and right to left pIC, as well as dense axonal fibers from both aIC and pIC at the nucleus accumbens core (NAc, Fig. 3d,e). Taken together, this anatomical study shows that although alC neurons project to different amygdala nuclei, the BLA remains the main target.

\section{Neurons of the IC monosynaptically excite BLA and CeM neurons}

To test the existence of a direct (monosynaptic) connection from insular neurons onto amygdala neurons we performed optogenetic circuit mapping of insular synaptic inputs onto BLA and CeM neurons using whole-cell patchclamp recordings ${ }^{17,30}$. We injected AAV9-CaMKII -ChR2-eYFP in the IC to record optically evoked excitatory and inhibitory postsynaptic currents (oEPSC and oIPSC) in neurons in amygdala nuclei clamped at $70 \mathrm{mV}$ and $0 \mathrm{mV}$, respectively (2 ms light pulse, Fig. $4 \mathrm{a}$ and Extended data Fig. 2b). First, we found that BLA neurons have higher membrane capacitance and lower membrane resistance compared to CeM neurons, consistent with the larger size of most BLA neurons which are excitatory, while most CeM neurons are smaller and inhibitory (Extended data Fig. 2a). Second, we observed in both BLA and CeM neurons a remaining fraction of the oEPSC after blockade of network activity (TTX+4AP, Fig. 4b,c), indicating monosynaptic excitatory inputs from the IC on both BLA and CeM neurons. The addition of glutamatergic antagonists (AP5+NBQX) abolished the monosynaptic excitatory response, confirming its glutamatergic nature (Fig. 4b,c). Third, olPSCs were systematically present, and abolished after TTX+4AP application, confirming they are polysynaptic connections as we used CaMKII promoter to express ChR2 in insular neurons. Finally, the latencies of polysynaptic oEPSC and oIPSC peak and monosynaptic oEPSC (TTX+4AP) from the onset of each light pulse were similar between IC-BLA and IC-CeM projection neurons (Fig. 4d and Extended data Fig. 2c). Interestingly, the latency from light onset to the oPSC peak was shorter for the oEPSC than for the oIPSC in both neuronal populations (Fig. 4d), in line with their respective mono- and poly-synaptic nature. Together, these results demonstrate that neurons of the IC glutamatergic neurons mono- and poly-synaptically excite BLA and CeM neurons, and polysynaptically recruit local inhibition.

\section{IC-BLA and IC-CeM synapses exhibit different short-term dynamics}

In order to examine the release properties of insular presynaptic synapses in different downstream neurons, we used paired- or train-pulse stimulation protocols ${ }^{31}$. Paired-pulse photostimulation of insular terminals ( $2 \mathrm{~ms}$ light pulse, $50 \mathrm{~ms}$ of interstimulus interval) was applied to measure excitatory and inhibitory pairedpulse ratios (PPRs) in BLA or CeM neurons (Fig. 4e). The PPRs of oEPSC and oIPSC were $<1$, indicating that insular inputs on BLA and CeM neurons are depressing (Fig. 4e). Although PPRs were 
similar in BLA and CeM neurons, train stimulations (10 pulses of $2 \mathrm{~ms}, 50 \mathrm{~ms}$ interval) of insular terminals revealed that, starting from the third photostimulation, IC inputs to BLA neurons were more depressed than IC inputs to CeM neurons (Fig. 4f,g).

\section{alC-BLA and BLA-alC recurrent connections}

Reciprocal connection between the IC and BLA has been described ${ }^{32}$. However, the recurrent nature of this loop had not been explored. Using a combination of retrograde tracing and optogenetic circuit mapping, we identified the existence of a recurrent circuit with monosynaptic excitation of BLA-aIC neurons by alC inputs, as well as monosynaptic excitation of alC-BLA neurons by BLA inputs (Fig. 4h,i).

\section{IC-BLA and IC-CeM neurons have distinct intrinsic properties}

Intrinsic membrane properties of IC-BLA and IC-CeM neurons were recorded from neurons labelled with retrograde tracers (Fig. 4j-m and Extended data Fig. 2d,e). Interestingly, the membrane capacitance of ICBLA was larger than the membrane capacitance of ICCeM projection neurons, suggesting IC-BLA neurons are larger compared to ICCeM neurons (Fig. 4n). Other passive membrane properties, such as membrane resistance and input resistance, were comparable between these two types of projection neurons (Fig. 4n and Extended data Fig. 2d). Some active properties were also different between the two neuronal projection populations, including the firing threshold, which was markedly higher in ICBLA neurons compared to ICCeM neurons (Fig. 40), as well as the firing frequency induced by current injection which was different between these two projection populations (Fig. 4p). Interestingly, the firing frequency was higher in IC-CeM neurons for low injected currents, whereas injection of larger currents induced a higher firing frequency in ICBLA neurons. Overall, these data suggest that insula neurons have different electrical and synaptic properties depending on their projection target, suggesting they might support different functions.

\section{aIC-BLA projection neurons control anxiety and are more active in anxiogenic spaces}

As we observed that glutamatergic neurons of the aIC control the level of anxiety (Fig. 1), and their main downstream target is the BLA (Fig. 3), we hypothesized that alC-BLA neurons are a major contributor to this function. To test the causal role of aIC-BLA projection neurons in anxiety-related behaviors, we used an optogenetic approach during anxiety assays, using the novel opsin somBiPOLES ${ }^{33}$. This somatargeted opsin is a fusion protein of the inhibitory opsin GtACR2 ${ }^{34}$ and the excitatory opsin Chrimson ${ }^{35}$, enabling activation and inhibition of the same neuronal populations through illumination at different wavelengths (Fig. 5a-c). We expressed somBiPOLES bilaterally, in alC-BLA neurons through a dual viral vector approach, and manipulated their activity through optic fibers implanted above the aIC (Extended data Fig. 3a,b). To evaluate the instantaneous effect of activation or inhibition of alC-BLA neurons on anxietyrelated behaviors, mice were tested in sessions composed of 6 epochs, beginning with neural activation (orange light), followed by inhibition (blue light) and a resting epoch (OFF, Fig. 5d,e). Averaged 
over all epochs (activation/inhibition/OFF), the time spent in the anxiogenic zone, was lower for the somBiPOLES group, which spent less time in the center of the OFT and tended to spend less time in the open arms of the EPM ( $p=0.23)$, in comparison to the control group (mCerulean, Fig. 5d,e). Importantly, no effect of light was detected on locomotion, as measured by distance travelled in the OFT (Fig. 5f). After behavioral tests, alC-BLA neurons were illuminated with orange light (activation of Chrimson), and immunofluorescent staining of cFos in fixed brain slices revealed a significant increase of cFos expressing cells in somBiPOLES expressing neurons compared to control neurons expressing mCerulean (Extended data Fig. 3c). Taken together, our results support that aICBLA neurons play a functional role in anxietyrelated behaviors. Nevertheless, as both activation and inhibition of alCBLA projections neurons decrease anxiety-related behavior, these causal experiments do not provide information on how aIC-BLA neurons encode anxiety.

Thus, we used fiber photometry, by expressing GCaMP6m selectively in aIC-BLA neurons by using a credependent dual-virus strategy, and implanting an optical fiber in the aIC (Fig. 5g,h and Extended data Fig. $4 a, b)$ to record calcium signals (Fig. 5i,j) during anxiety-related behaviors. While the mice explored the EPM (Fig. 5k), the global calcium signal was increased in the open arms compared to the closed arms (Fig. 5l and Extended Video 1), and the frequency of calcium transients tended to increase in the open arms ( $p=0.12$, Fig. $5 \mathrm{~m})$. In the OFT, we also observed an increase of the global calcium signal in the anxiogenic space (center, Fig. 5o,p), as well as a trend for an increased in the frequency of calcium transients $(p=0.16$, Fig. $5 q)$.

To test the link between calcium signal of aIC-BLA projection neurons and trait anxiety, we correlated the difference between the calcium transients frequency in anxiogenic and safe spaces, with the overall anxiety level of individual animals, estimated by the percentage of time spent in the open arms of the EPM. We reasoned that the most anxious mice spent the least time in the open arms. Interestingly, for the transients recorded in the EPM, but not the OFT, the differential transient frequency (open-closed) is positively correlated with the anxiety level of the animals (Fig. $5 n, r$ ), linking the transient activity of alCBLA neurons in anxiogenic spaces to the animal level of trait anxiety. Altogether, our data show that the activity of alCBLA neurons controls the level of state anxiety, is increased in anxiogenic spaces, and is correlated to trait anxiety.

\section{Bidirectional representation of valence in alC-BLA projection neurons}

Using optogenetic real-time place preference, a previous study has shown that the aIC-BLA pathway drives place preference, suggesting this pathway contributes to code for positive valence ${ }^{27}$. To bidirectionally test the causal role of aIC-BLA projection neurons in valence-related behaviors, we used a cre-dependent dual viral strategy to express either the excitatory opsin ChR2 or the inhibitory opsin GtACR2 in alC-BLA neurons, and implanted a fiber optic over the alC (Extended data Fig. 5a,b). After confirmed that illumination of alC-BLA neurons expressing GtACR2 induces an inhibition of action potential firing in these neurons using whole-cell patch-clamp recordings ex vivo (Extended data Fig. 5ce), 
we tested the impact of activation or inhibition of aIC-BLA neurons in a closed-loop realtime place preference/avoidance assay (RTPP/A). In this test, mice freely explored two chambers, including one where alCBLA projection neurons were activated or inhibited, depending on the opsin expressed.

Photoactivation of alC-BLA projection neurons only tended to induce a preference for the light-paired side, compared to control mice ( $p=0.07$, Fig. $6 a, b)$, while photoinhibition of this same population, in another group of mice, induced a preference for the light-paired side compared to control mice (Fig. 6c,d). Together, these data show that inhibition of aICBLA projection neurons can induce place preference, which suggests these neurons are involved in negative valence.

To identify how aIC-BLA neurons encode emotional valence, we performed fiber photometry recordings of this population during valence-related behaviors. Interestingly, during sucrose consumption, we observed a decrease of the calcium signal after mice licked the sucrose solution (Fig. $6 \mathrm{f}, \mathrm{g}$ ). In contrast, quinine consumption did not alter calcium signals after licking the solution (Fig. 6i,j). However, mild foot-shocks (10 shocks, 0.3 mA, 1 s duration, Fig. 6k) strongly increased aIC-BLA calcium signal (Fig. 6l,m). Finally, a third stimulation of negative valence (tail suspension Fig. $6 \mathrm{n}$ ) also induced an increase of the calcium signal in alC-BLA neurons (Fig. 6o,p). Together, these data suggest that aIC-BLA neurons bidirectionally encode valence through an inhibition in response to positive valence and activation in response to negative valence.

\section{Discussion}

Our study reveals the alC as a common brain substrate encoding both valence and anxiety-related behaviors. We show that aIC projection neurons, and specifically aIC-BLA neurons are more active in anxiogenic spaces, and that aIC-BLA neurons are activated by aversive stimuli.

In vivo calcium imaging using fiber photometry showed that glutamatergic neurons in the alC are more active in anxiogenic spaces, suggesting the activity of alC excitatory neurons is anxiogenic. We confirmed the anxiogenic property of this neural population as chemogenetic inhibition of alC glutamatergic neurons induced an increase in the time spent in the anxiogenic zone, which is consistent with previous pharmacological experiments ${ }^{15}$. Recent work also identified a contribution of the pIC in processing aversive states and anxiety-related behaviors ${ }^{13}$. Interestingly, this study described a decrease of pIC glutamatergic neurons activity when mice where located in the open arms of elevated mazes placed in a high anxiety setting (minimal handling, new room and high light intensity). In the 'low-anxiety' conditions we used, including handling, room habituation, low red-light intensity, we observed stable pIC neuronal activity in the EPM, which is consistent with the model predicting that pIC excitatory neurons can shift behavioral strategies upon the detection of aversive internal states ${ }^{13}$. Consistently with these studies, we demonstrate the anteroposterior dichotomy of the IC control of anxiety-related behaviors, with an anxiogenic function of the activity of aIC glutamatergic neurons, which represents anxiogenic spaces.

Excitatory neurons of the $\mathrm{IIC}$ and pIC have been shown to differentially control emotional valence, especially for tastants ${ }^{14}$. Using fiber photometry recordings of excitatory neurons, we found a selective 
increase of pIC excitatory neurons activity after quinine consumption, whereas, no changes in neural activity were observed after sucrose consumption in either aIC or pIC. These results are consistent with two-photon calcium imaging data where pIC neurons respond specifically to a bitter taste ${ }^{36}$. Our results are also consistent with studies describing a spatially distributed representation of tastants in the alc ${ }^{37}$, but contrast with the selective coding of sweet tastants in the alC described by others ${ }^{36}$. However, the latest included recordings of all neurons, only in the superficial layers of the IC, whereas we include all layers and record only glutamatergic neurons. This suggests that sweet tastants could be differentially encoded by excitatory and inhibitory cell types, and/or cortical layers in the alC. Together, these results suggest a functional role of pIC neurons in negative valence processing, while the contribution of alC glutamatergic neurons in positive valence remains unclear.

Seminal and modern studies highlighted the amygdala as a downstream target of the insula $24,26,27,38,39$. Among the 12 regions of interest we assessed with anterograde mapping, we found that amygdala nuclei (BLA, CeL and CeM) receive the strongest projection from both anterior and posterior insular cortices. However, our data challenge the existence of two segregated insula-amygdala pathways (aIC-BLA and pIC-CeA $)^{27}$, as we also found substantial alC-CeA and pIC-BLA projections. We confirmed the existence of functional monosynaptic excitatory inputs from the IC onto BLA and CeM neurons, along with recruitment of polysynaptic inhibition. Since the BLA is mainly composed of excitatory neurons, the polysynaptic inhibition might be through feedforward and/or feedback inhibition. On the other hand, as most CeM neurons are inhibitory, the polysynaptic inhibition in this region is likely supported by feedforward inhibition. Interestingly, we observed short-term synaptic depression in both insula-BLA and insula-CeM synapses, with a stronger depression in insula-BLA than insula-CeM synapses. Finally, we identified different intrinsic properties of insula-BLA and insula-CeM neurons, also supporting a model where these two pathways underlie divergent functions.

Using optogenetic tool for both neuronal excitation and inhibition (somBiPOLES) ${ }^{33}$, we revealed an anxiogenic effect of alC-BLA manipulation, without real-time effects of neural activation or inhibition in the EPM and OFT. Indeed, the average time spent in the center of the OFT is decreased by the alteration of aICBLA neural activity, independently of type of manipulation (activation, inhibition, no manipulation) and the same trend was observed in the EPM. Thus, alteration of alC-BLA neuronal activity appears to increase anxiety-related behaviors, although this effect is not instantaneous. This data suggest that anxiety-related information is carried by an extended neuronal network including aIC-BLA projection neurons, rather than in an isolated pathway.

Neural recordings showed that the calcium signal in alC-BLA neurons is increased in the anxiogenic zone of the EPM and OFT. In addition, we found that in the aICBLA neurons, the difference of transients in open-closed arms is positively correlated with the anxiety level of the animals, estimated by the time spent in the open arms of the EPM, which support the involvement of this neuronal population in trait anxiety. The implication of aIC-BLA neurons in the control of anxiety-related behaviors and their increased activity in anxiogenic spaces suggest that aIC-BLA neurons encode anxiogenic spaces in real-time, which 
will alter anxiety-related behaviors on a longer time scale, in the order of tens of minutes. This hypothesis is consistent with a model proposing that insular neurons code information at multiple time scales ${ }^{40,41}$. Together, these results show that aIC-BLA neurons control anxiety-related behaviors and encode anxiogenic spaces.

To assess the causal role of aIC-BLA neurons in emotional valence, we used optogenetics in real-time place preference/aversion assays. Interestingly, inhibition of aIC-BLA neurons drives place preference, while activation only induces a trend for a preference. Whereas these results could appear conflicting, as both activation and inhibition of the same neuronal population would induce a similar behavioral outcome, this could also imply that activation and inhibition of aIC-BLA neurons both alter the coding properties of the manipulated neurons, leading to similar effects. Thus, to disentangle how alC-BLA neurons encode valence-related behavior, we recorded their neuronal activity using fiber photometry. Our data show bidirectional coding properties of aIC-BLA neurons which are inhibited by stimuli of positive valence and excited by stimulation of negative valence. In conclusion, our study reveals aIC-BLA projection neurons as a crucial building block of the neural circuit linking anxiety- and valence-related behaviors.

Clinical and preclinical studies report both the insula and amygdala as key brain regions involved in several psychiatric disorders, including anxiety disorders as well as addictions, which are both characterized by disruption of valence assessment ${ }^{11,13,42-44}$. Patients with anxiety disorders present an attentional bias for stimuli of negative valence ${ }^{7}$, while in drug addiction, the attribution of emotional valence to drug-related cues participates to relapse in humans and animal models ${ }^{45-47}$. Thus, our findings provide a starting point for further characterize the role of insulaamygdala pathways in psychiatric disorders including addiction and anxiety disorders.

\section{Declarations}

\section{Acknowledgements}

The authors acknowledge the entire Beyeler Lab, Nadine Gogolla and Christian Lüscher for insightful discussions and advice. We thank the Bordeaux Imaging Center (BIC) for the use of their confocal microscope. We thank Sara Laumond, Julie Tessaire and the technical staff of the animal housing facility of the Neurocentre Magendie (INSERM 1215) for their invaluable support.

\section{Funding}

We acknowledge the support of the Région Nouvelle-Aquitaine, the INSERM Avenir program of the French $\mathrm{NIH}$ (INSERM), the Fondation NRJ-Institut de France to the Beyeler Lab, and of the Brain and Behavior Research Foundation NARSAD young investigator grant to $A B$ and Fondation pour la Recherche Médicale fellowship, (FRM, ARF201909009147) to CN.

\section{Authorship Contributions}

Page $11 / 24$ 
CN, AJ, WY, SD, HE, LS, CF, AV, MM, DJ, AB carried out the experiments, CN, AJ, SD, HE, WY, LS performed data analysis. $C N, A J, W Y, S D$ and $A B$ designed the study. SW and SRR provided the viral vectors for the somBiPOLES experiment. CN, AJ and AB wrote the manuscript. All authors critically reviewed the content and approved the final version before submission.

\section{Competing interests}

The authors declare that they do not have any competing interests or conflicts of interest (financial or otherwise) related to the material presented in this manuscript.

\section{References}

1. American Psychiatric Association. Diagnostic and Statistical Manual of Mental Disorders (DSM-5®). (American Psychiatric Pub, 2013).

2. Daviu, N., Bruchas, M. R., Moghaddam, B., Sandi, C. \& Beyeler, A. Neurobiological links between stress and anxiety. Neurobiology of Stress 11, 100191 (2019).

3. Steimer, T. The biology of fear- and anxiety-related behaviors. Dialogues Clin Neurosci 4, 231-249 (2002).

4. Belzung, C. \& Griebel, G. Measuring normal and pathological anxiety-like behaviour in mice: a review. Behav. Brain Res. 125, 141-149 (2001).

5. Sailer, U. et al. Altered reward processing in the nucleus accumbens and mesial prefrontal cortex of patients with posttraumatic stress disorder. Neuropsychologia 46, 2836-2844 (2008).

6. Fox, E., Yates, A. \& Ashwin, C. Trait Anxiety and Perceptual Load as Determinants of Emotion Processing in a Fear Conditioning Paradigm. Emotion 12, 236-249 (2012).

7. MacLeod, C., Grafton, B. \& Notebaert, L. Anxiety-Linked Attentional Bias: Is It Reliable? Annu Rev Clin Psychol 15, 529-554 (2019).

8. Paulus, M. P. \& Stein, M. B. An insular view of anxiety. Biol Psychiatry 60, 383-387 (2006).

9. Klumpp, H., Fitzgerald, D. A. \& Phan, K. L. Neural predictors and mechanisms of cognitive behavioral therapy on threat processing in social anxiety disorder. Prog. Neuropsychopharmacol. Biol. Psychiatry 45, 83-91 (2013).

10. Klumpp, H., Angstadt, M. \& Phan, K. L. Insula Reactivity and Connectivity to Anterior Cingulate Cortex When Processing Threat in Generalized Social Anxiety Disorder. Biol Psychol 89, 273-276 (2012).

11. Etkin, A. \& Wager, T. D. Functional neuroimaging of anxiety: a meta-analysis of emotional processing in PTSD, social anxiety disorder, and specific phobia. Am J Psychiatry 164, 1476-1488 (2007).

12. Shah, S. G., Klumpp, H., Angstadt, M., Nathan, P. J. \& Phan, K. L. Amygdala and insula response to emotional images in patients with generalized social anxiety disorder. J Psychiatry Neurosci 34, 296-302 (2009).

13. Gehrlach, D. A. et al. Aversive state processing in the posterior insular cortex. Nat Neurosci 22, 14241437 (2019). 
14. Peng, Y. et al. Sweet and bitter taste in the brain of awake behaving animals. Nature $527,512-515$ (2015).

15. Méndez-Ruette, M. et al. The Role of the Rodent Insula in Anxiety. Front. Physiol. 10, (2019).

16. Pichon, S., Miendlarzewska, E. A., Eryilmaz, H. \& Vuilleumier, P. Cumulative activation during positive and negative events and state anxiety predicts subsequent inertia of amygdala reactivity. Soc Cogn Affect Neurosci 10, 180-190 (2015).

17. Felix-Ortiz, A. C. et al. BLA to vHPC Inputs Modulate Anxiety-Related Behaviors. Neuron 79, 658-664 (2013).

18. Tye, K. M. et al. Amygdala circuitry mediating reversible and bidirectional control of anxiety. Nature 471, 358-362 (2011).

19. Beyeler, A. et al. Divergent Routing of Positive and Negative Information from the Amygdala during Memory Retrieval. Neuron 90, 348-361 (2016).

20. Kim, J., Pignatelli, M., Xu, S., Itohara, S. \& Tonegawa, S. Antagonistic negative and positive neurons of the basolateral amygdala. Nat Neurosci 19, 1636-1646 (2016).

21. Namburi, P. et al. A circuit mechanism for differentiating positive and negative associations. Nature 520, 675-678 (2015).

22. Baur, V., Hänggi, J., Langer, N. \& Jäncke, L. Resting-State Functional and Structural Connectivity Within an Insula-Amygdala Route Specifically Index State and Trait Anxiety. Biological Psychiatry 73, 85-92 (2013).

23. Jung, Y.-H. et al. Altered Amygdala Resting-State Functional Connectivity and Hemispheric Asymmetry in Patients With Social Anxiety Disorder. Front. Psychiatry 9, 164 (2018).

24. Gehrlach, D. A. et al. A whole-brain connectivity map of mouse insular cortex. eL ife 9, e55585 (2020).

25. Schiff, H. C. et al. An Insula-Central Amygdala Circuit for Guiding Tastant-Reinforced Choice Behavior. J. Neurosci. 38, 1418-1429 (2018).

26. Shi, C. J. \& Cassell, M. D. Cortical, thalamic, and amygdaloid connections of the anterior and posterior insular cortices. J. Comp. Neurol. 399, 440-468 (1998).

27. Wang, L. et al. The coding of valence and identity in the mammalian taste system. Nature $558,127-$ 131 (2018).

28. Ju, A., Fernandez-Arroyo, B., Wu, Y., Jacky, D. \& Beyeler, A. Expression of serotonin $1 A$ and $2 A$ receptors in molecular- and projection-defined neurons of the mouse insular cortex. (2020) doi:10.21203/rs.2.20095/v3.

29. Griebel, G. \& Holmes, A. 50 years of hurdles and hope in anxiolytic drug discovery. Nat Rev Drug Discov 12, 667-687 (2013).

30. Petreanu, L., Huber, D., Sobczyk, A. \& Svoboda, K. Channelrhodopsin-2-assisted circuit mapping of long-range callosal projections. Nat Neurosci 10, 663-668 (2007).

31. Citri, A. \& Malenka, R. C. Synaptic Plasticity: Multiple Forms, Functions, and Mechanisms. Neuropsychopharmacol 33, 18-41 (2008). 
32. Morceau, S., Piquet, R., Wolff, M. \& Parkes, S. L. Targeting Reciprocally Connected Brain Regions Through CAV-2 Mediated Interventions. Front Mol Neurosci 12, 303 (2019).

33. Vierock, J. et al. BiPOLES is an optogenetic tool developed for bidirectional dual-color control of neurons. Nat Commun 12, 4527 (2021).

34. Govorunova, E. G., Sineshchekov, O. A., Janz, R., Liu, X. \& Spudich, J. L. NEUROSCIENCE. Natural light-gated anion channels: A family of microbial rhodopsins for advanced optogenetics. Science 349, 647-650 (2015).

35. Klapoetke, N. C. et al. Independent Optical Excitation of Distinct Neural Populations. Nat Methods 11, 338-346 (2014).

36. Chen, X., Gabitto, M., Peng, Y., Ryba, N. J. P. \& Zuker, C. S. A gustotopic map of taste qualities in the mammalian brain. Science 333, 1262-1266 (2011).

37. Chen, K., Kogan, J. F. \& Fontanini, A. Spatially Distributed Representation of Taste Quality in the Gustatory Insular Cortex of Behaving Mice. Current Biology 31, 247-256.e4 (2021).

38. Reep, R. L. \& Winans, S. S. Efferent connections of dorsal and ventral agranular insular cortex in the hamster, Mesocricetus auratus. Neuroscience 7, 2609-2635 (1982).

39. Saper, C. B. Convergence of autonomic and limbic connections in the insular cortex of the rat. J. Comp. Neurol. 210, 163-173 (1982).

40. Livneh, Y. et al. Estimation of Current and Future Physiological States in Insular Cortex. Neuron 105, 1094-1111.e10 (2020).

41. Livneh, Y. \& Andermann, M. L. Cellular activity in insular cortex across seconds to hours: Sensations and predictions of bodily states. Neuron (2021) doi:10.1016/j.neuron.2021.08.036.

42. Naqvi, N. H., Rudrauf, D., Damasio, H. \& Bechara, A. Damage to the insula disrupts addiction to cigarette smoking. Science 315, 531-534 (2007).

43. Nicolas, C. et al. Longitudinal Changes in Brain Metabolic Activity after Withdrawal from Escalation of Cocaine Self-Administration. Neuropsychopharmacology 42, 1981-1990 (2017).

44. Venniro, M. et al. The Anterior Insular Cortex $\rightarrow$ Central Amygdala Glutamatergic Pathway Is Critical to Relapse after Contingency Management. Neuron 96, 414-427.e8 (2017).

45. Grimm, J. W., Hope, B. T., Wise, R. A. \& Shaham, Y. Neuroadaptation. Incubation of cocaine craving after withdrawal. Nature 412, 141-142 (2001).

46. Nicolas, C. et al. Incubation of Cocaine Craving After Intermittent-Access Self-administration: Sex Differences and Estrous Cycle. Biol Psychiatry 85, 915-924 (2019).

47. O’Brien, C. P., Childress, A. R., McLellan, A. T. \& Ehrman, R. Classical conditioning in drug-dependent humans. Ann N Y Acad Sci 654, 400-415 (1992).

\section{Figures}



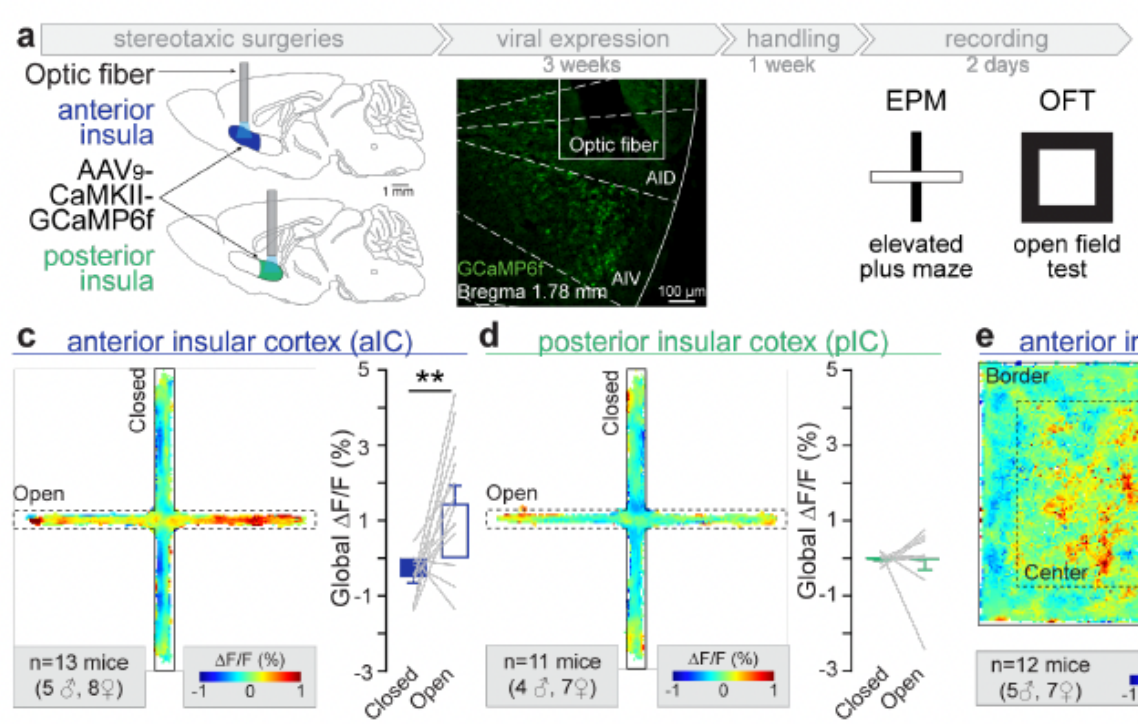

b Calcium signal in glutamatergic projection neurons

d

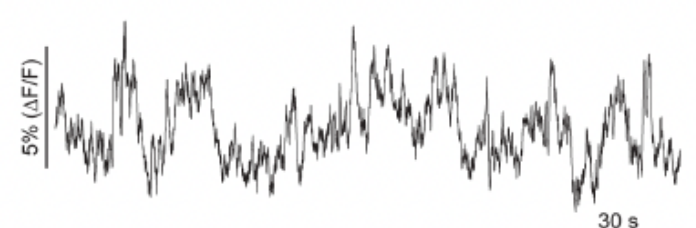

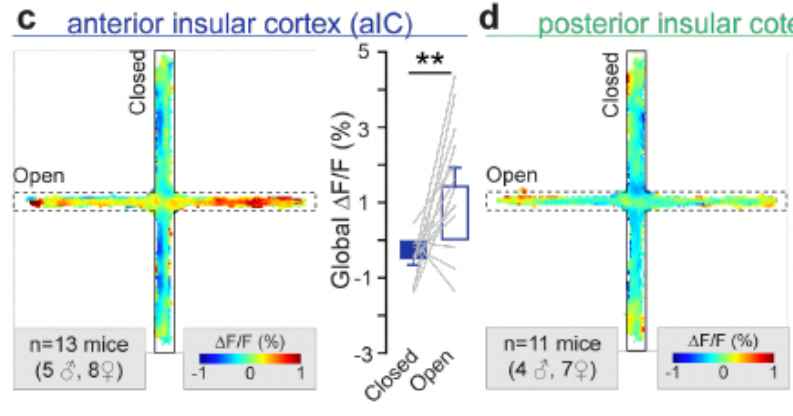
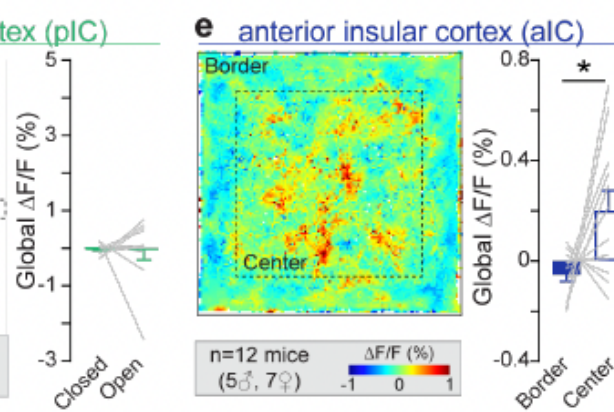

f posterior insular cotex (p|C)

g viral expression behavioral test day 1

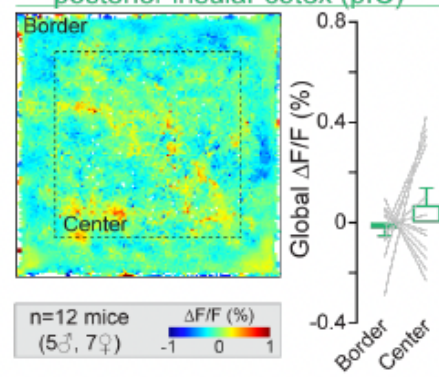

g stereotaxic surgeries
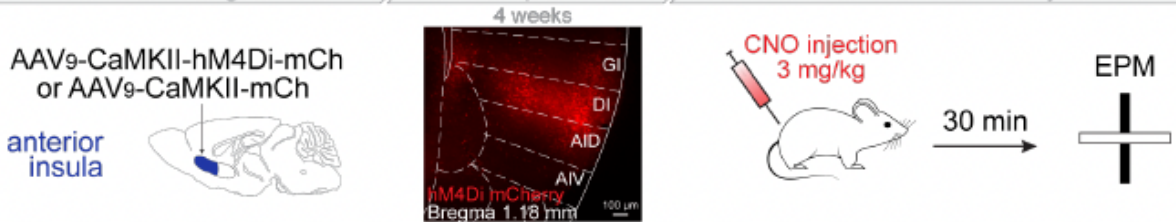

behavioral test day 2
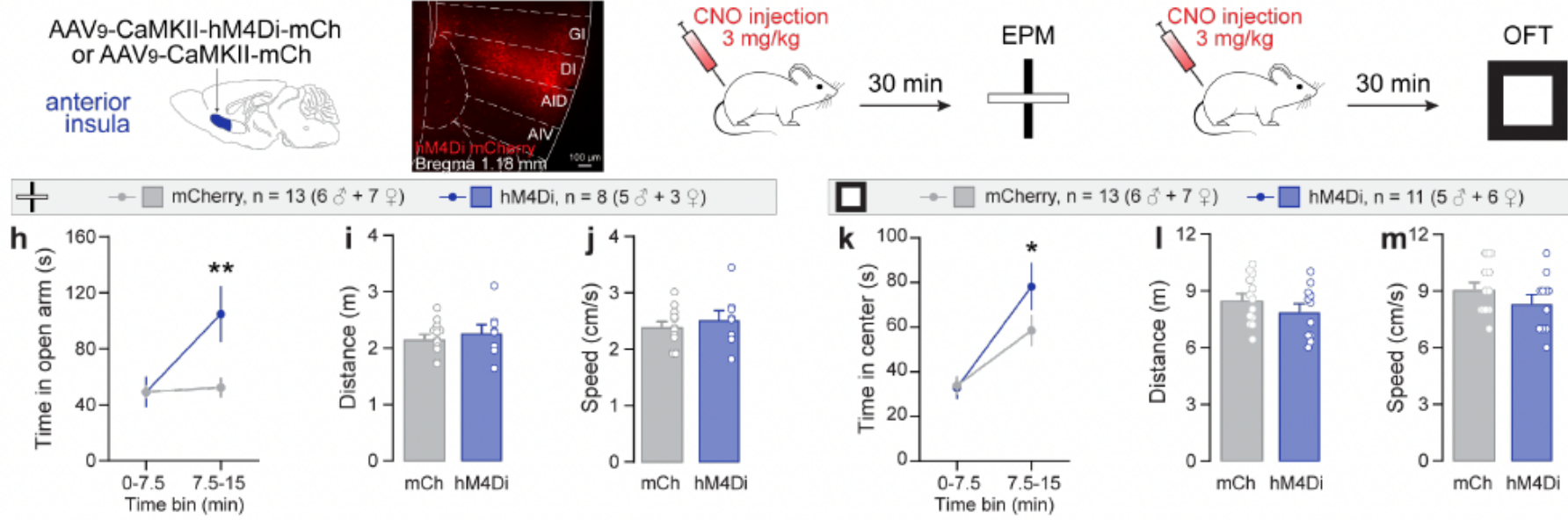

\section{Figure 1}

Anxiety-related activity in aIC and pIC glutamatergic neurons. a. Experimental scheme of viral strategy to express GCaMP6f, and fiber implantation over the insula followed by 3 weeks of recovery and viral expression. One week of handling precedes the recording session in both EPM and OFT. b. Bulk GCaMP6f signal recorded from alC glutamatergic neurons. $\Delta \mathrm{F} / \mathrm{F}$ represents the fluorescent changes from the mean level of entire recording time series. c. Averaged heat map of global calcium signal recorded from alC neurons during 15 min EPM test. Global calcium signal is increased in open compared to closed arms (Two-tailed paired t-test, $* * p=0.0048, n=13$ ). $d$. Averaged heat map of global calcium signal recorded from pIC during EPM test $(n=11)$. Global calcium signal is similar in the open and closed arms. e. Averaged heat map of global calcium signal recorded from alC neurons during OFT test. Global calcium signal is increased in the center compared to the border (Two-tailed paired $t$ test, ${ }^{*} \mathrm{p}=0.0334, \mathrm{n}=12$ ). $\mathrm{f}$. Averaged heat map of global calcium signal recorded from pIC neurons during OFT test $(n=12)$. Global calcium signal is similar in the center and the border. g. Experimental timeline. Viral strategy to express hM4Di in alC glutamatergic neurons followed by 4 weeks of recovery and viral expression. Mice received a CNO injection $(3 \mathrm{mg} / \mathrm{kg}) 30$ minutes before the EPM and OFT tests. $\mathrm{h}$. Time spent in the open arms is 
increased in hM4Di group compared to control mCherry $(\mathrm{mCh})$ group during the second time bin (7.5-15 $\mathrm{min}$ ) of the EPM test (Two-way ANOVA, time: $F(1,19)=13.43$, $* \star p=0.0016$, virus: $F(1,19)=3.585$, $p=0.0736$, time $x$ virus: $F(1,19)=10.73, * * p=0.004$, post hoc Fischer's LSD for time bin (7.5-15): $\star * p=0.0026, m C h n=13, h M 4 D i n=8)$. i. Locomotion in $E P M$, as distance travelled in meters is similar in hM4Di $(n=8)$ and $\mathrm{mCh}(\mathrm{n}=13)$. j. Average speed in $\mathrm{cm} / \mathrm{s}$ during EPM test is similar between hM4Di $(n=8)$ and mCh $(n=13)$. $k$. Time spent in the center of the OFT test is increased in hM4Di group compared to the control $\mathrm{mCh}$ during the second time bin (7.5-15 min) (Two-way ANOVA, time: $F(1,22)=54.25$, $* \star \star p<$ 0.0001 , virus: $F(1,22)=1.196, p=0.2860$, time $x$ virus: $F(1,22)=4.769,{ }^{*} p=0.0399$, post hoc Fischer's LSD for time bin (7.5-15): * $\mathrm{p}=0.049, \mathrm{mCh} n=13, \mathrm{hM} 4 \mathrm{Di} \mathrm{n}=11)$. I. Locomotion in OFT, as distance travelled in meters is similar in hM4Di $(n=11)$ and $m C h(n=13)$. m. Average speed in $\mathrm{cm} / \mathrm{s}$ during the OFT test is similar between hM4Di $(n=11)$ and $m C h(n=13)$. All the results are represented as mean \pm SEM.
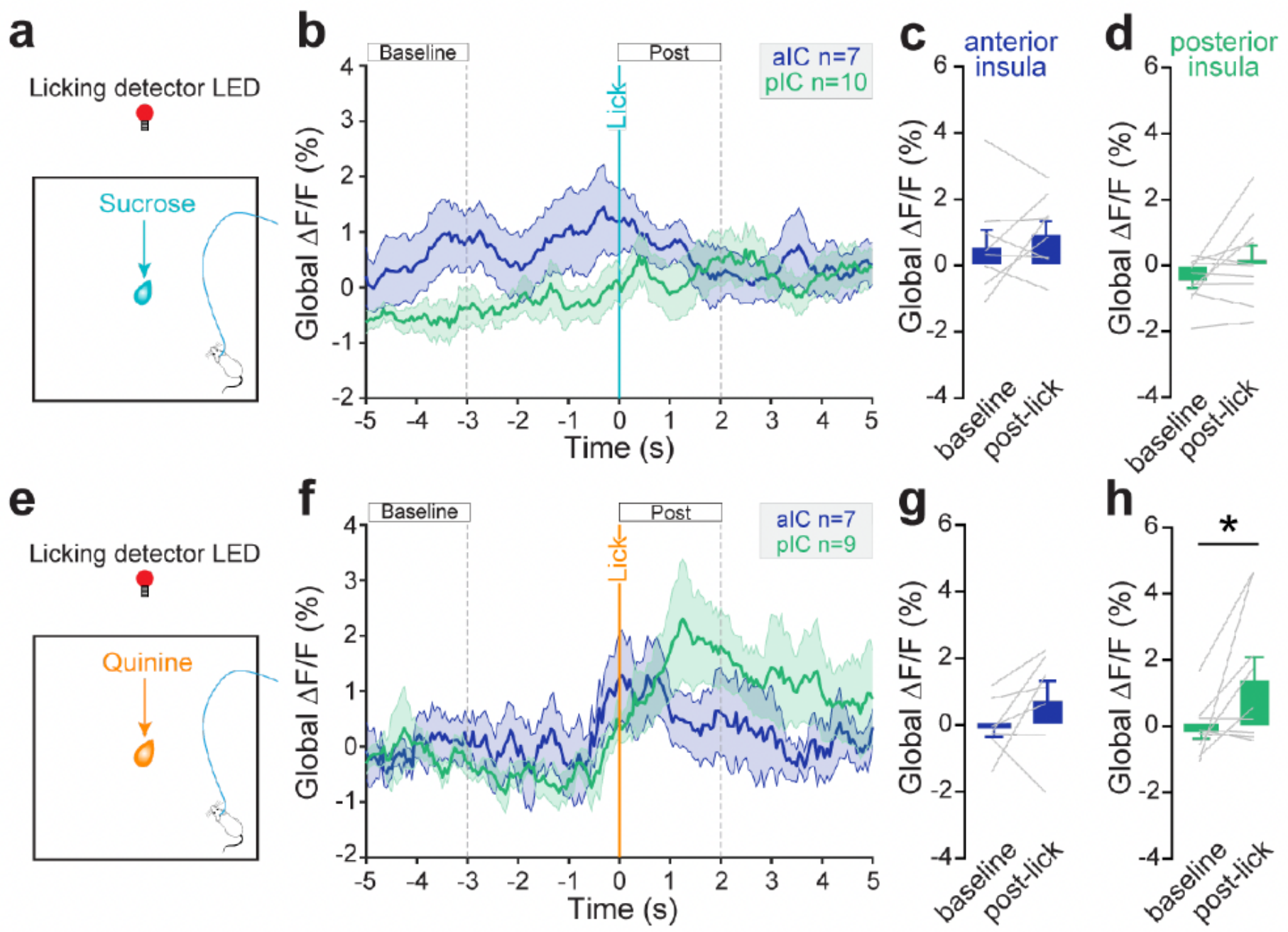

\section{Figure 2}

Valence-related activity in aIC and pIC glutamatergic neurons. a. Schematic of sucrose consumption test. b. Peri-licking analysis of the calcium signal between baseline and sucrose post-lick in the alC and pIC (alC $n=7, p l C n=10)$. c. Bar plot of global calcium signal during baseline and sucrose post lick in alC 
glutamatergic neurons (alC $n=7)$. d. Bar plot of global calcium signal during baseline and sucrose post lick in plC glutamatergic neurons ( $\mathrm{plC} n=10$ ). e. Schematic of quinine consumption test. f. Peri-licking analysis of the calcium signal between baseline and quinine post-lick in the alC and pIC (aIC n=7, pIC $\mathrm{n}=10) \mathrm{g}$. Bar plot of global calcium signal during baseline and quinine post lick in pIC glutamatergic neurons ( $p I C n=9$ ). d. Global calcium signal increased during quinine post lick in pIC glutamatergic neurons compared to baseline (Two tailed paired $t$ test, ${ }^{*} p=0.0486, p I C n=9$ ). All the results are represented as mean \pm SEM.
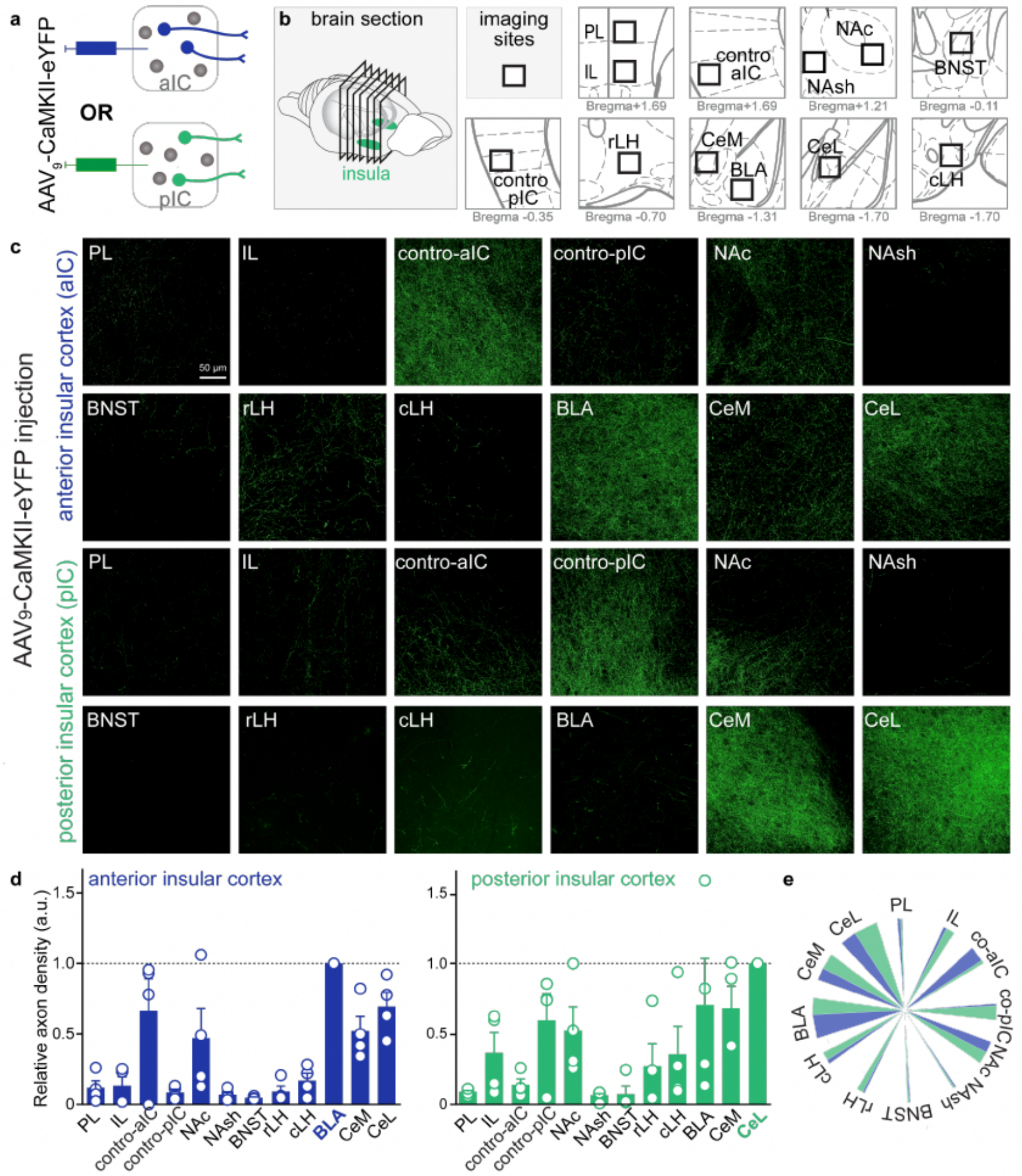


\section{Figure 3}

Downstream regions of aIC and pIC projection neurons. a-b. Experimental scheme of viral expression (a) and imaging downstream regions (b), including prelimbic (PL), infralimbic (IL), contralateral aIC (controaIC), contralateral pIC (contro-pIC), nucleus accumbens core (NAc), nucleus accumbens shell (NAsh), bed nucleus of the stria terminals (BNST), rostral lateral hypothalamus (rLH), caudal lateral hypothalamus (CLH), basolateral amygdala (BLA), medial (CeM) and lateral (CeL) subdivision of central amygdala. c. Representative image of eYFP+ axonal projections from one mouse expressing CaMKII eYFP in neurons of aIC (top) or pIC (bottom). d. Number of fluorescent pixels normalized to the average value of the maximal projection region (BLA for aIC and CeL for pIC) per image from aIC and pIC groups. e. Summary pie chart of relative fluorescent intensity in projecting regions from alC (blue) and pIC (green). All the results are represented as mean \pm SEM. 

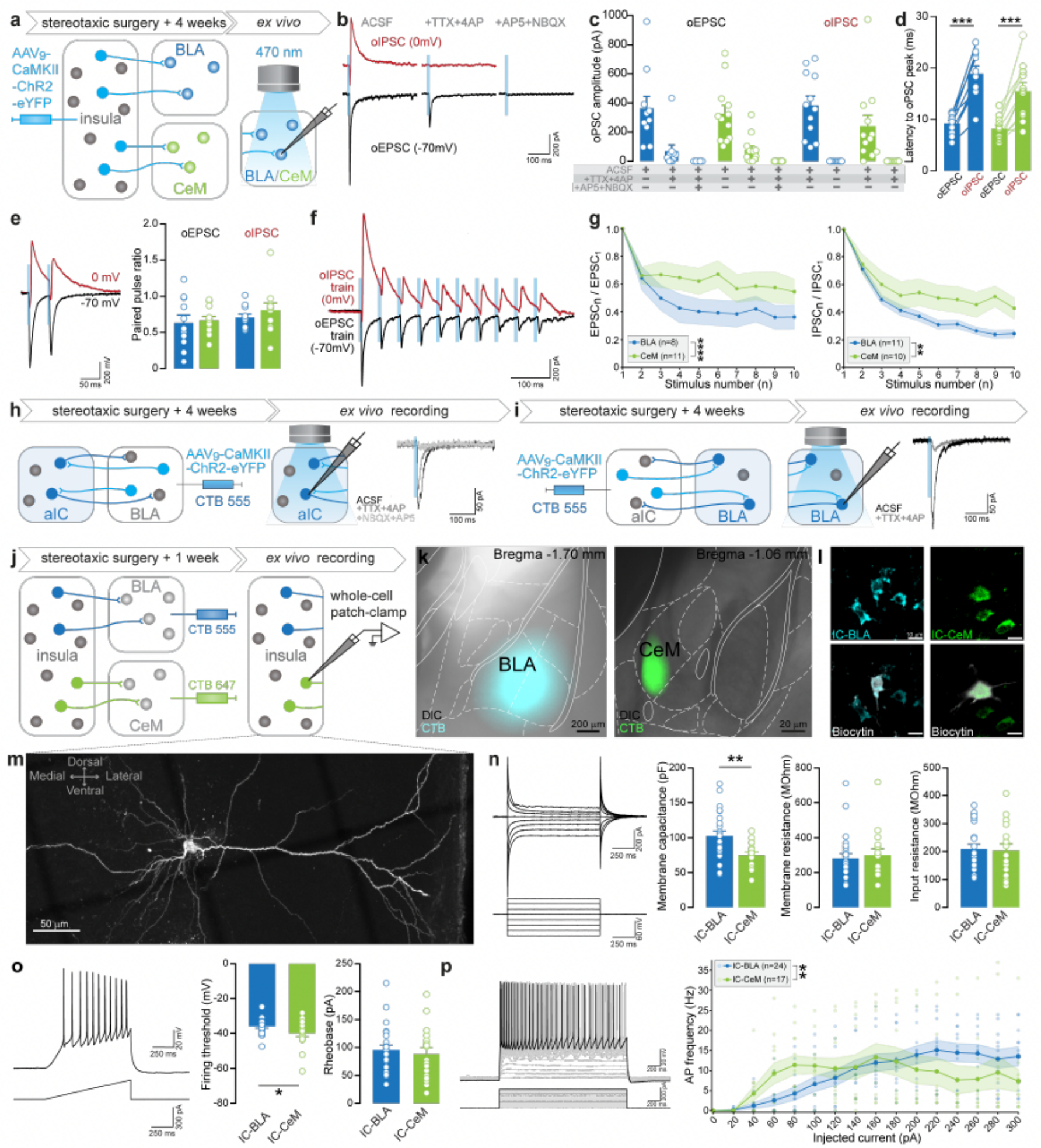

\section{Figure 4}

Properties of IC-BLA and IC-CeM synapses, and of IC-BLA and IC-CeM neurons. a. Experimental strategy of expressing ChR2-eYFP in the insular neurons to confirm the monosynaptic connection of the insular neurons to basolateral amygdala (BLA, IC-BLA) and medial subdivision of central amygdala (CeM, ICCeM) and record the dynamic properties of insular synapses on BLA and CeM synapses upon the optogenetic activation of the insular terminals. b. Representative traces of synaptic responses upon the 
application of ACSF, + TTX+4AP and +AP $5+N B Q X$ in order to prove the monosynaptic connection of the insular neurons to the BLA and CeM neurons. c. Quantification of optogenetic-induced excitatory/inhibitory postsynaptic currents (oEPSC/oIPSC) of BLA and CeM neurons upon optogenetic activations of insular axonal terminals during the application of ACSF, +TTX+4AP and +AP5+NBQX. $d$. Latency to the peak of oEPSC or oIPSC during the application of ACSF (Two-tailed paired t-test $\star \star \star * p<0.0001$ for $B L A n=10, * \star * p=0.0003$ for CeM $n=11$ ). e. Representative traces and summary data of paired pulse ratio at $-70 \mathrm{mV}$ (excitatory PPR) and $0 \mathrm{mV}$ (inhibitory PPR) at the IC-BLA and IC-CeM synapses. (Two-way ANOVA, drug: $F(1,23)=42.24$, ${ }^{* \star} p<0.0001$, BLA $n=12$, CeM $\left.n=10\right)$. f-g. Representative traces (f) and summary data $(\mathrm{g})$ of excitatory or inhibitory responses of BLA and CeM neurons upon the 10 train stimulations of ChR2 in the insular terminals. h-i. Experimental scheme and representative oEPSC traces of the IC-BLA neurons after the stimulation of the BLA terminals (h) and the BLA-IC neurons after the stimulation of the insular terminals (i) during ACSF, +TTX+4AP and +AP5+NBQX applications in order to prove the reciprocal connections between the insula and BLA. j. Experimental plan for CTB labeling and whole-cell patch recording of IC-BLA and IC-CeM. $k$. Representative images of CTB injection sites in BLA and CeM. I. Representative images of biocytin-filled neurons labelled by CTB. m. Confocal image of biocytin-filled neurons in the insula. n. Membrane capacitance and resistance of IC-BLA and IC-CeM neurons measured in voltage-clamp mode. Input resistance of those neurons was acquired from current voltage curve (IC-BLA n=24, IC-CeM n=17). o. Firing threshold and rheobase of IC-BLA and IC CeM neurons, measured in current clamp mode (IC-BLA $n=24, I C-C e M n=17)$. p. Action potential frequency versus injected current of IC-BLA and IC-CeM projection neurons measured in current clamp (IC-BLA $n=24$, IC-CeM $n=17$ ). All the results are represented as mean \pm SEM. 

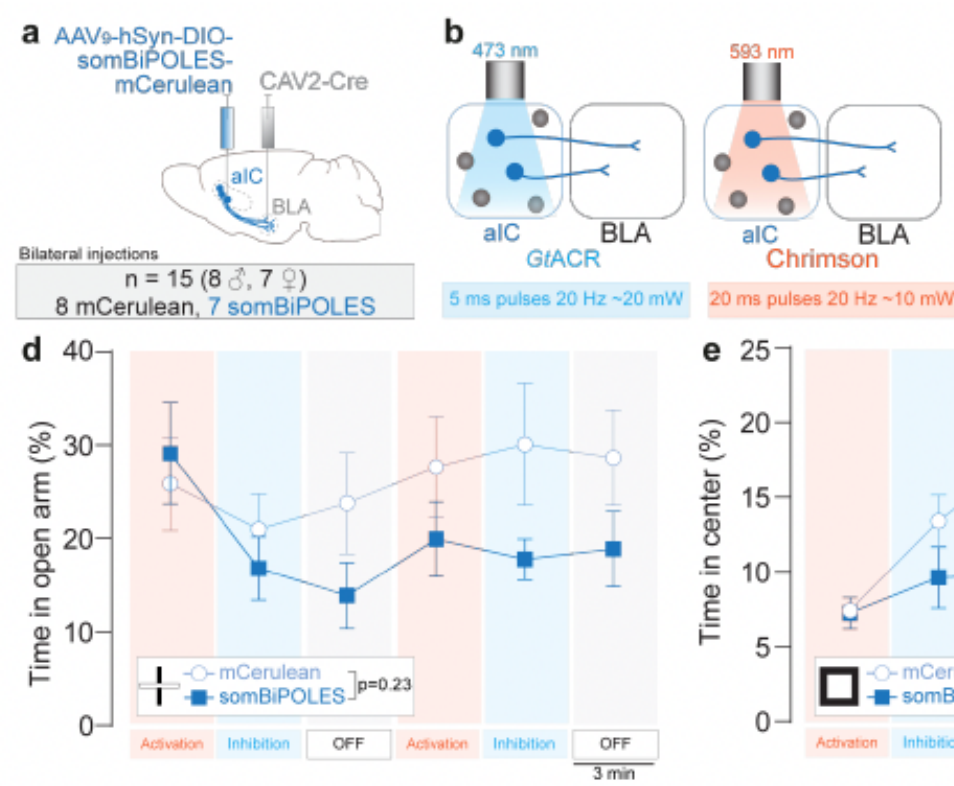

c
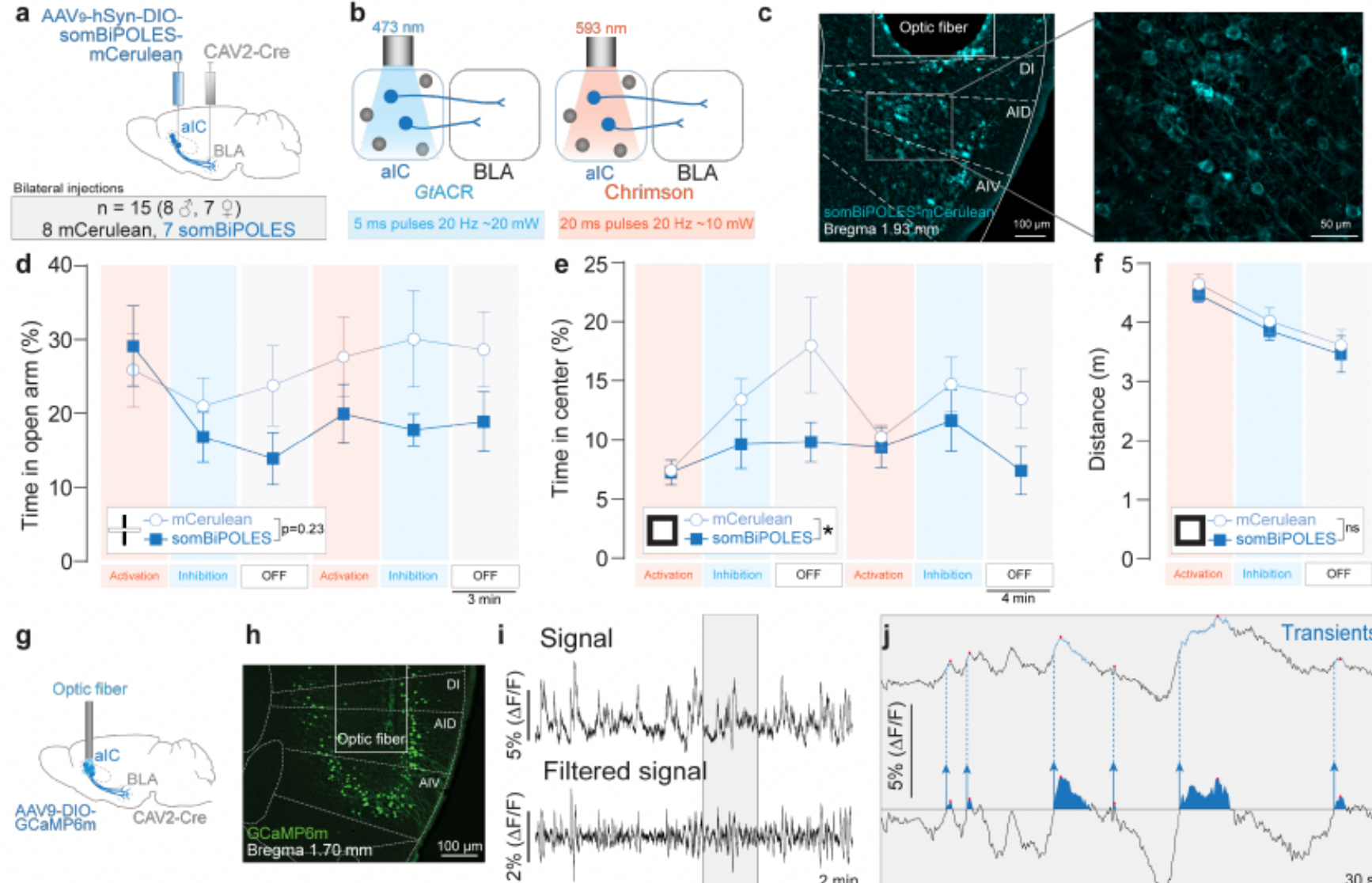

i Signal
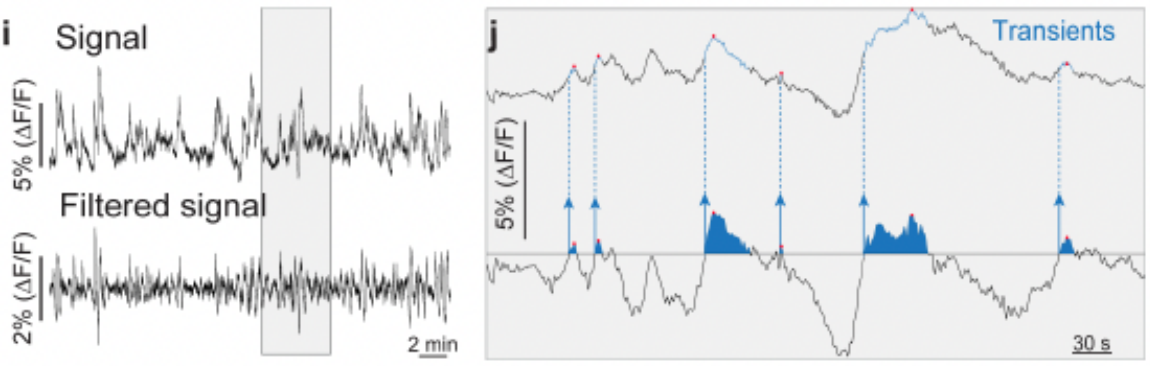

k

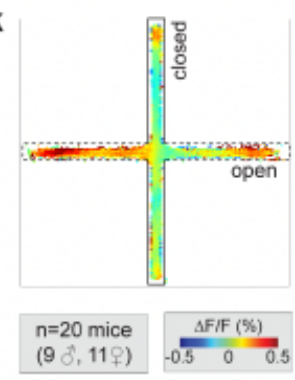

- Border

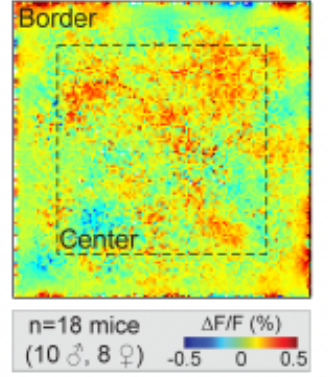

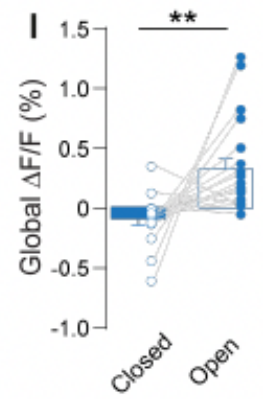

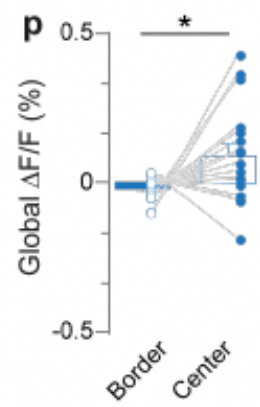

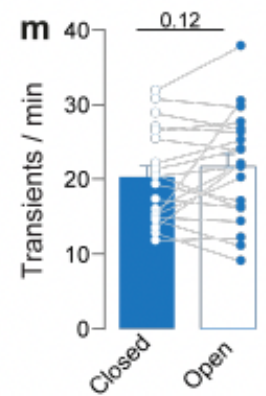

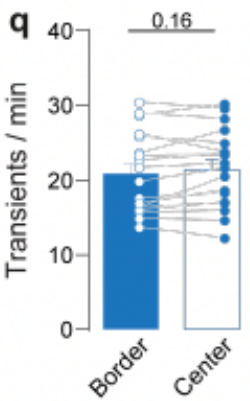

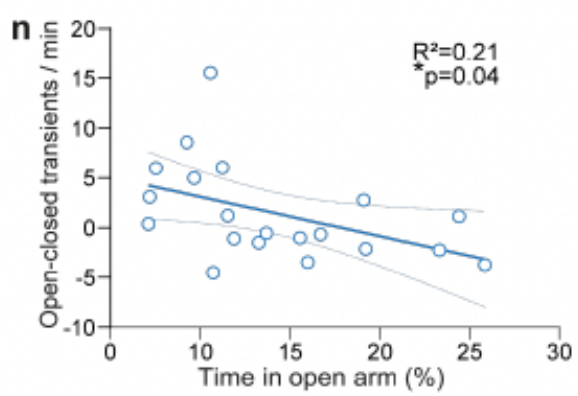

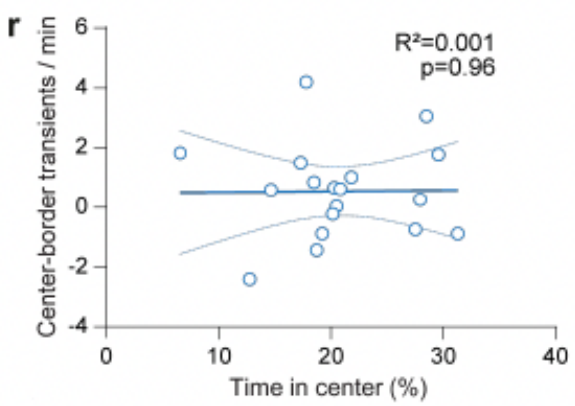

\section{Figure 5}

Role of aIC-BLA neurons during anxiety-related behaviors. a. Experimental scheme of injection of the viral vector carrying somBiPOLES (or Flex.mCerulean) opsin in alC, expressed in cell bodies and CAV2-Cre injected in BLA. b. Scheme of somBiPOLES experimental manipulation, in which blue light $(473 \mathrm{~nm})$ is used to inhibit neuronal population through the soma-targeted GtACR component of somBiPOLES, while orange $(593 \mathrm{~nm}$ ) light is used to stimulate neuronal population through the Chrimson component of 
somBiPOLES. c. Representative image of coronal sections of aIC, expressing cell bodies of somBiPOLES along with fiber placement. d. Percentage of time spent in open arms of EPM, across the 6 epochs of 3 mins. Independently of the stimulation epoch procedure, somBiPOLES group has a trend to spend less time in open arms compared to control mCerulean (Two-way ANOVA, time: $F(3.061,39.79)=2.28, p=0.09$, opsin: $F(1,13)=1.596, p=0.228$, time $x$ opsin: $F(5,65)=1.610, p=0.170$, somBiPOLES $n=7$, mCerulean $n=8)$. e. Percentage of time spent in the center of OFT, across each epoch. Independently of the stimulation epoch procedure, somBiPOLES group spent less time in the center compared to control mCerulean (Twoway ANOVA, time: $F(2.583,33.58)=2.792, p=0.06$, opsin: $F(1,13)=5.632,{ }^{*} p=0.03$, time $x$ opsin: $F(5,65)=1.132, p=0.35$, somBiPOLES $n=7$, mCerulean $n=8)$.). $f$. Locomotion in open field test, as distance travelled in the arena in meters, epochs are averaged. (Two-way ANOVA, time: $F(1.728,22.47)=19.63$, ${ }^{*} p<0.001$, opsin: $F(1,13)=0.454, p=0.51$, time $x$ opsin: $F(2,26)=0.003, p=0.997$, somBiPOLES $n=7$, mCerulean $\mathrm{n}=8)$. g. Strategy for recording neuronal activity from aIC-BLA neurons in wild-type mice. $\mathrm{h}$. Representative images of GCaMP6m expression in alC. i. Fiber photometry signal recorded from alC BLA neurons, (Top) Bulk GCaMP6m signal, and (Bottom) filtered GCaMP6m signal for calcium transients' detection. $\Delta F / F$ represents the fluorescent changes from the mean level of the entire recording time series. j. Representation of automated transients' detection. Filtered GCaMP6m peaks exceeding the threshold (horizontal line in the lower trace) were identified as the transients. k. Averaged heat map of global calcium signal recorded from aIC-BLA neurons during EPM test. I. Global calcium signal is increased in the open arms compared to the closed arms (Two-tailed paired t-test, ${ }^{*} \mathrm{p}=0.003, \mathrm{n}=20$ ). $\mathrm{m}$. Calcium transients frequency is similar in open and closed arms $(n=20)$. $n$. Calcium transients frequency (open-closed) difference correlates with mice spent time in open arms (linear regression: $F(1,18)=4.903$, $\left.\mathrm{R} 2=0.2141,{ }^{*} \mathrm{p}=0.040, \mathrm{n}=20\right)$. $\mathrm{o}$. Averaged heat map of global calcium signal recorded from alC-BLA neurons during OFT test $(n=18)$. p. Global calcium signal is increased in the center compared to the border (Two-tailed paired t-test, ${ }^{*} \mathrm{p}=0.021, \mathrm{n}=18$ ). q. Calcium transients' frequency is similar in the center and the border $r$. Correlation of calcium transients' frequency (center-border) difference and time spent in the center. All the results are represented as mean \pm SEM. 

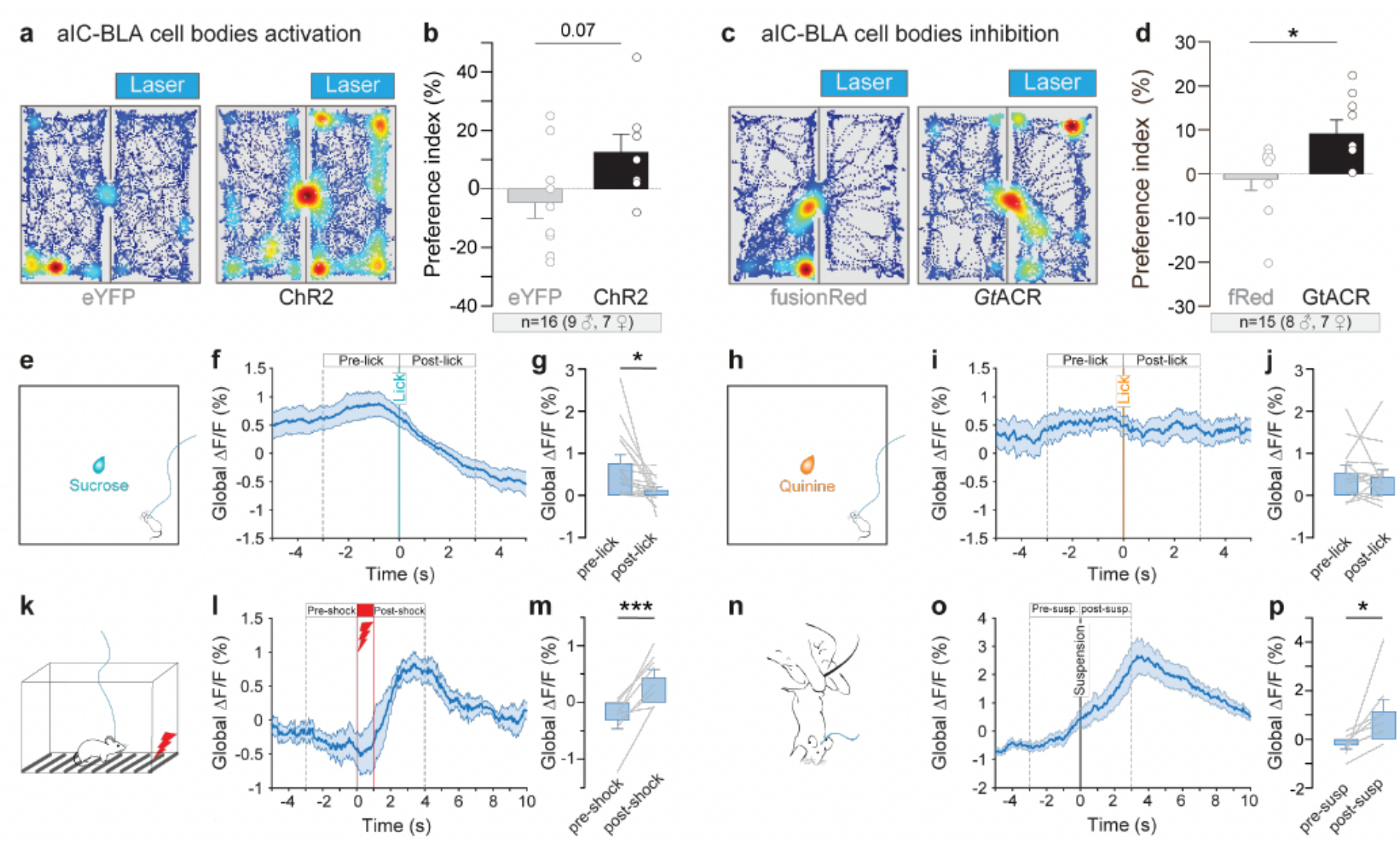

\section{Figure 6}

Role of aIC-BLA neurons in valence-related behaviors. a. Two representative traces showing an occupancy heatmap of the time spent in the non-stimulated (left) or stimulated (right) side for a control eYFP mouse (left) and ChR2 mouse (right). b. Preference index calculated as the time difference between the stimulation side and non-stimulation side, divided by total time spent and multiplied by 100 . ChR2 group tends to spend more time on the stimulation side in comparison to the control group (Two-tailed unpaired t-test, $p=0.074$, eYFP $n=7$, ChR2 $n=7$ ). c. Two representative traces showing an occupancy heatmap of time spent in the non-stimulated (left) or stimulated (right) side for a control fusionRed mouse (left) and GtACR mouse (right). d. Preference index is increased in GtACR group where alC-BLA glutamatergic neurons are inhibited compared to the fRed control group (Two-tailed unpaired t-test, ${ }^{*} p=0.035$, fRed $n=8, G t A C R n=7$ ). e. Schematic of sucrose consumption test. f. Peri-licking analysis of the calcium signal between sucrose pre- and post-lick. g. Global calcium signal during sucrose post-lick is decreased compared to pre-lick (Two-tailed paired t-test, ${ }^{*} p=0.028, n=11$ ). h. Schematic of quinine consumption test. i. Peri-licking analysis of the calcium signal between quinine pre- and post-lick. j. Bar plot of global calcium signal during quinine pre and post lick $(n=9)$. $k$. Schematic of the foot-shock test. I. Peri-event analysis of the calcium signal between pre- and post-shock $(n=8) . m$. Global calcium signal is increased during post-shock compared to pre-shock (Two-tailed paired t-test, ${ }^{* \star *} p=0.0006, n=8$ ). $n$.

Schematic of the tail suspension test. 0 . Peri-event analysis of the calcium signal between pre-and posttail suspension $(n=8)$. p. Global calcium signal is increased during post-tail suspension compared to pretail suspension (Two-tailed paired t test, ${ }^{*} \mathrm{p}=0.0272, \mathrm{n}=8$ ). All the results are represented as mean $\pm \mathrm{SEM}$. 


\section{Supplementary Files}

This is a list of supplementary files associated with this preprint. Click to download.

- NicolasJuetalaICBLAEPM.mp4

- NicolasJuetalSOM.pdf 